\title{
Chromosomal Instability and Telomere Shortening in Long-Term Culture of Hematopoietic Stem Cells: Insights from a Cell Culture Model of RPS14 Haploinsufficiency
}

\author{
K. Thomay ${ }^{a}$ A. Schienke ${ }^{a} \quad$ B. Vajen ${ }^{a} \quad$ U. Modlich $^{b} \quad$ A. Schambach ${ }^{b}$ \\ W. Hofmann ${ }^{\text {a }}$ B. Schlegelberger ${ }^{a} \quad$ G. Göhring ${ }^{a}$
}

Institutes of a Cell and Molecular Pathology, and ${ }^{b}$ Experimental Hematology, Hannover Medical School, Hannover, Germany

\section{Key Words}

Cell culture $\cdot$ Chromosomal instability $\cdot$ Hematopoietic stem cells $\cdot$ Myelodysplastic syndrome $\cdot$ Telomeres

\begin{abstract}
The fate of cultivated primary hematopoietic stem cells (HSCs) with respect to genetic instability and telomere attrition has not yet been described in great detail. Thus, knowledge of the genetic constitution of HSCs is important when interpreting results of HSCs in culture. While establishing a cell culture model for myelodysplastic syndrome with a deletion in $5 q$ by performing RPS14 knockdown, we found surprising data that may be of importance for any CD34+ cell culture experiments. We performed cytogenetic analyses and telomere length measurement on transduced CD34+ cells and untransduced control cells to observe the effects of long-term culturing. Initially, CD34+ cells had a normal median telomere length of about $12 \mathrm{~kb}$ and showed no signs of chromosomal instability. During follow-up, the median telomere length seemed to decrease and, simultaneously, increased chromosomal instability could be observed - in modified and control cells. One culture showed a clonal monosomy 7 - independent of prior RPS14 knockdown. During further culturing, it seemed that the telomeres re-elon-
\end{abstract}

gated, and chromosomes stabilized, while TERT expression was not elevated. In summary, irrespective of our results of RPS14 knockdown in the long-term culture of CD34+ cells, it becomes clear that cell culture artefacts inducing telomere shortening and chromosomal instability have to be taken into account and regular cytogenetic analyses should always be performed.

(c) 2013 S. Karger AG, Basel

Cultivation of stem cells as well as induced pluripotent stem cells continues to be a challenge. However, due to constantly expanding knowledge about cytokines and niche conditions, it is now possible to cultivate hematopoietic stem cells (HSCs) in short-term culture for about 2 weeks and in long-term culture with appropriate feeder layers for more than 6 weeks, both with ongoing differentiation. Long-term cultivation is known to harbor the risk of genetic instability [Moon et al., 2011; Ross et al., 2011; Prockop and Keating, 2012]. However, the fate of cultivated primary hematopoietic stem cells with respect to genetic instability and telomere attrition has not yet been described in much detail. Thus, knowledge on the genetic constitution of HSCs is important when interpreting results of HSCs in culture [Lefort et al., 2009; Ge et al., 2011].

\section{KARGER}

E-Mail karger@karger.com

www.karger.com/cgr
C) 2013 S. Karger AG, Basel

$1424-8581 / 13 / 1421-0014 \$ 38.00 / 0$
PD Dr. Gudrun Göhring

Institute of Cell and Molecular Pathology, Hannover Medical School Carl-Neuberg-Strasse 1

DE-30625 Hannover (Germany)

E-Mail goehring.gudrun@mh-hannover.de 
Originally, the aim of our experiments was to create a cell culture model to analyze the myelodysplastic syndrome (MDS) with an isolated deletion in $5 \mathrm{q}$, a neoplasia derived from hematopoietic stem cells and a distinct subtype of MDS associated with a favorable prognosis. Ebert et al. [2008] identified RPS14 coding for a ribosomal protein as the candidate gene in MDS with del $(5 q)$, since haploinsufficiency of RPS14 leads to an erythroid differentiation defect corresponding to the anemia observed in patients with MDS and $\operatorname{del}(5 \mathrm{q})$. We showed earlier that leukemic progression is associated with telomere shortening, chromosomal instability, clonal evolution, and the development of complex karyotypes [Göhring et al., 2010, 2012; Jädersten et al., 2011]. However, further studies were required to better understand the mechanisms responsible for disease progression.

Unfortunately, it is almost impossible to keep patientderived MDS cells in long-term culture. So-called MDS cell lines have been shown to have already transformed into an aggressive leukemic state [Drexler et al., 2009]. Thus, so far no cell culture model is available to gain mechanistic insights into the pathogenesis of MDS. We reasoned that these limitations may be overcome by the development of a long-term culture (LTC) model of human HSCs, the CD34+ cells, with shRNA-mediated downregulation of RPS14 to the level of haploinsufficiency.

While putting modified and control HSCs in culture for several weeks, we performed cytogenetic analyses and telomere length measurement to observe the effects of long-term culturing. Surprisingly, we found evidence of telomere shortening in modified and control cells and the development of a clone with stable chromosome aberration in a culture of control cells. These findings may be of importance to more precisely interpret the results of HSCs in culture. Thus, the emphasis of this paper lies on the importance of performing regular cytogenetic analyses in HSC culture experiments so that valuable research work does not get distracted by cell culture artefacts.

\section{Materials and Methods}

\section{Isolation of CD34+ Cells}

CD34+ cells were isolated from umbilical cord blood of healthy donors via Ficoll gradient centrifugation followed by Magnetic Cell Separation (Miltenyi, Germany) to a purity of $>90 \%$. The study of CD34+ cells was approved by the Ethics Committee of Hannover Medical School (No. 835-2010). Umbilical cord blood was donated after written informed consent by women after childbirth at Hannover Medical School.

Chromosomal Instability and Telomeres in CD34+ Cell Culture
Lentiviral Knockdown of RPS14

RPS14 haploinsufficiency was achieved via transduction (MOI 8) of lentiviral shRNA vectors (shRNA1 and shRNA2) as described previously [Ebert et al., 2008]. The vectors used in the previous study were modified replacing the PURO cassette with an eGFPpre-cassette from pRRL.PPT.SF.GFPpre that was kindly provided by A. Schambach (Hannover Medical School). As controls, untransduced CD34+ cells (mock cells) and scrambled shRNA (Plasmid 17920, Addgene)-transduced CD34+ cells were used. Two days after transduction, the successfully transduced cells were sorted for the internal marker GFP via fluorescence-activated cell sorting and used for measuring expression levels and performing longterm culture.

\section{Expression Analyses}

After lentivirally induced RPS14 knockdown, relative RPS14 and TERT levels on the RNA and protein level were obtained via real-time PCR, using B2M as internal standard, and Western Blot (using anti-RPS14 (H130, sc 68873) antibody 1:500, Santa Cruz) analysis according to standard procedures in CD34+ cells and K562 cells, respectively.

\section{Long-Term Culture}

Before irradiation, M2-10B4 were grown in RPMI (Biochrom) $+10 \%$ FCS (FBS Superior, Biochrom). During transduction and sorting processes, CD34+ cells were grown in Stem Span and cytokines (hFlt3 $100 \mathrm{ng} / \mathrm{ml}$; hSCF $100 \mathrm{ng} / \mathrm{ml}$; hTPO 20 ng/ml; hIL-6 $20 \mathrm{ng} / \mathrm{ml}$, Peprotech). The Magnetic Cell Separation and fluorescence-activated cell sorting-sorted RPS14-deficient CD34+ cells were grown for at least 6 weeks on irradiated murine feeder layers (80 Gy) that constitutively secrete human growth factors (M210B4, CLS Cell Lines Service) [Hogge et al., 1996] according to standard procedures in MyeloCult (StemCell Technologies). For optimal growth of CD34+ cells on irradiated M2-10B4, hydrocortisone $\left(10^{-6} \mathrm{M}\right.$, StemCell Technologies) and cytokines (hFlt3 $20 \mathrm{ng} /$ $\mathrm{ml}$; hSCF 20 ng/ml; hTPO 4 ng/ml; hIL-6 4 ng/ml, Peprotech) were added [Hogge et al., 1996; Tehranchi et al., 2010]. On a weekly basis, half the medium was changed and, if possible regarding the cell numbers, half the cells were taken for experiments. Due to the limitation of cell numbers, follow-up experiments were performed - if possible - on a 2-week basis.

In total, 4 independent long-term culture sets (performed at subsequent time points) were established, composed of primary hematopoietic stem cells transduced with either shRNA1, shRNA2 or scrambled shRNA, and from mock cells (i.e. $4 \times 4$ cultures analyzed for 6-7 weeks). Proliferation was estimated via the cell number.

\section{Differentiation Assay}

The differentiation ability was assessed after $0,2,4$ and 6 weeks in long-term culture via a colony-forming cell assay according to standard procedures (MethoCult H4435, StemCell Technologies). Each experiment was performed in duplicate $(35 \mathrm{~mm}$ Petri dish format) and analyzed by 2 different persons independently of each other.

\section{DNA Repair Capacity}

DNA damage and repair was analyzed after $0,1,3,5$ and 7 weeks in long-term culture via immunofluorescence and quantification of double-strand break foci ( $\gamma \mathrm{H} 2 \mathrm{AX}$ foci) according to stan- 
dard procedures (anti- $\gamma \mathrm{H} 2 \mathrm{AX}$ antibody, clone JBW301, Millipore). Mitomycin C (MMC), a DNA cross-linking agent, was used to induce double-strand breaks. After a $1 \mathrm{~h}$ incubation time with a final MMC concentration of $3 \mu \mathrm{g} / \mathrm{ml}$, cell culture medium was replaced and cells incubated for a further hour before the immunofluorescence procedure. From each culture and time point, foci of 50 randomly chosen cells were counted.

\section{Cytogenetic Investigations}

Chromosome preparation and fluorescence R-banding were performed after 0, 2, 4 and 6 weeks in long-term culture, as previously described [Schlegelberger et al., 1999]. Karyotypes were described according to the International System for Human Cytogenetic Nomenclature [ISCN, 2009].

\section{Telomere/Centromere-Fluorescence in situ Hybridization}

Ten metaphases per experiment were analyzed after 0, 2, 4 and 6 weeks in long-term-culture applying combined fluorescence Rbanding and T/C-FISH (DAKO Denmark A/S, Denmark) as described previously [Lange et al., 2010]. For telomere length measurement, the ISIS-Telomere module (Metasystems, Germany) was used.

\section{$L M-P C R$}

LM-PCR was performed as described previously [Schmidt et al., 2001]. DNA was digested with 2.5 U Tsp509I (New England BioLabs, UK). Primer extension was performed using the biotinylated primer lvLTR1: $5^{\prime}$-bioGAACCCACTGCT TAAGCCTCA-3' following the first PCR $\left(95^{\circ} \mathrm{C}\right.$ for $5 \mathrm{~min}, 95^{\circ} \mathrm{C}$ for $1 \mathrm{~min}, 55^{\circ} \mathrm{C}$ for $30 \mathrm{~s}, 68^{\circ} \mathrm{C}$ for $2 \mathrm{~min}$ for $30 \mathrm{cycles}, 68^{\circ} \mathrm{C}$ for $10 \mathrm{~min}$ ) using Extensor Hi-Fidelity PCR Master Mix (ABgene, Germany) and the following primers: lvLTR2 $5^{\prime}$-AGCTTGCCTTGAGTGCTTCA- $3^{\prime}$ and lvLTR3 $5^{\prime}$-AGTAGT GTGTGCCCGTCTGT-3'. The nested PCR was performed under identical conditions: primer-3 $5^{\prime}$-AGTGGCACAGCAGTTAGGACG-3' and primer- $45^{\prime}$-TCCATGCCTTGCAAAATGGCG-3'. PCR products were isolated by gel electrophoresis, purified using QIA quick Gel Extraction Kit (Qiagen) and sequenced after subcloning into TA cloning vector (Invitrogen). Recovered sequences were screened using the NCBI human genome database. Gene classification followed database records and PubMed literature.

\section{Results}

\section{RPS14-Deficient CD34+ Cells in Long-Term Culture}

Lentiviral downregulation of RPS14 in CD34+ primary hematopoietic stem cells from cord blood led to an expression level of approximately $40 \%$ compared to mock cells. Western Blot analyses in the cell line K562 confirmed RPS14 knockdown on the protein level after transduction with RPS14 shRNA vectors in comparison to scrambled shRNA transduced cells and unmodified (mock) cells. This demonstrated the effectiveness of the vector (fig. 1).

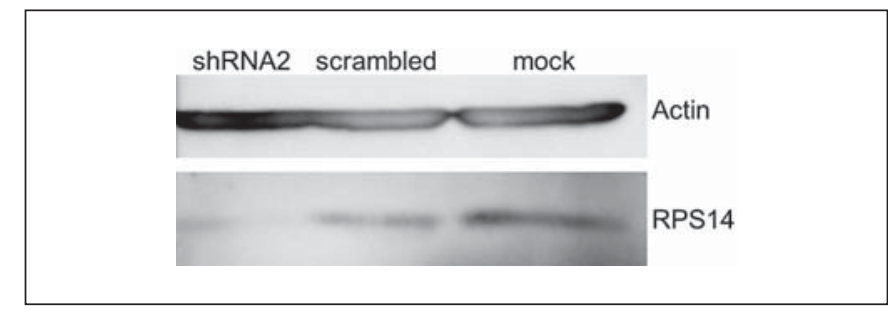

Fig. 1. Relative RPS14 protein in the cell line K562 after lentivirally induced knockdown of RPS14 (shRNA2) in comparison to scrambled shRNA-transduced cells and unmodified K562 cells (mock).

In all 4 LTC sets (i.e. shRNA1/2, scrambled and mock), the amount of cells increased over time. Generally, within 6 weeks, RPS14-deficient cells showed a poorer proliferation than the control cells with a 95-fold versus a 419fold multiplication, respectively.

\section{Differentiation Assay}

Colony-forming assays showed that, in comparison to the control cells, RPS14 haploinsufficiency immediately led to a decreased erythroid differentiation capacity. Most importantly, the percentage of erythroid progenitor colonies using cells at the start of the long-term culture (i.e. 2 days after transduction) was $11.6 \%$ in RPS14-deficient cells $(n=4)$ versus $32.9 \%$ in the control cells $(n=4)$. This erythroid differentiation defect is in line with previous data [Ebert et al., 2008] and increased even further during follow-up. With ongoing long-term culture, erythroid differention capacity decreased in all cells but, concurrent with the data at time point 0 , this effect was more pronounced in the RPS14-deficient cells (fig. 2).

Absolute colony numbers counted ranged between 14 and 90 (median: 52 colonies/plate) in assays of RPS14deficient cells and 10 and 173 (median: 49 colonies/plate) in control cells.

\section{DNA Damage in Long-Term Culture}

To analyze a possible role of aberrant DNA doublestrand break repair, we measured the number of $\gamma \mathrm{H} 2 \mathrm{AX}$ foci per cell. Median numbers of $\gamma \mathrm{H} 2 \mathrm{AX}$ foci after all time points measured were 9.5 for untreated RPS14-deficient cells ( $\mathrm{n}=13$ measurements from 4 independent LTC and 5 time points $(0,1,3,5$, and 7 weeks of LTC); in 7 cases, the cell number was too low for analysis). In the control cells, median foci number was 10.5 foci/cell $(\mathrm{n}=14)$. After MMC treatment, foci numbers increased to 14.6 ( $\mathrm{n}=$ $13)$ and 14.5 foci/cell $(\mathrm{n}=14)$ in RPS14-deficient cells and DOI: $10.1159 / 000356096$
Thomay et al. 


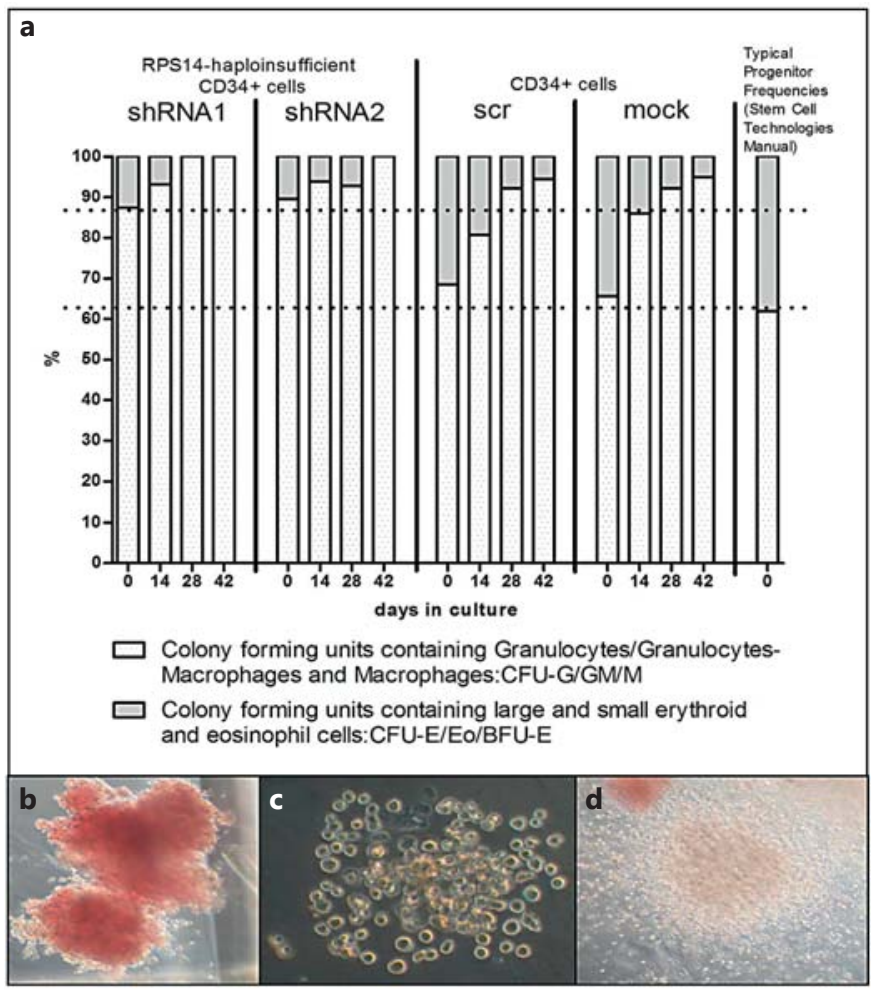

Fig. 2. a Progenitor frequencies in methylcellulose assays comparing RPS14-deficient cells (shRNA $1+2$ ) with control cells (scrambled and mock) after 0, 2, 4, and 6 weeks in long-term culture. Each bar comprises an average value of 4 replicates. b Erythroid colony: BFU-E (burst-forming unit-erythrocytes). c Macrophage colony: CFU-M (colony-forming unit-macrophages). d Neutrophil-granulocyte colony: CFU-G (colony-forming unit-neutrophils-granulocytes).

control cells, respectively. Thus, foci numbers show an (expected) increase in double-strand break after MMC treatment in RPS14-deficient and control cells. However, compared to the control cells, RPS14-deficient cells show no statistically relevant sign of increased sensitivity towards DNA damage at any time point (fig. 3 ).

\section{Cytogenetic Analyses in Long-Term Culture}

At day 0, all CD34+ cells showed a normal karyotype without chromosome breakage. After 2 weeks, we detected increased chromosome breakage (defined as at least one break/metaphase) (fig. 4a) in one of 4 LTCs from mock CD34+ cells (1 metaphase with a break in $1 \mathrm{p}, 1$ metaphase with a deletion in 3p, 1 metaphase with a deletion in 6q, 2 metaphases with a deletion in 7q). LTCs from RPS14-deficient cells $(\mathrm{n}=4)$ and scrambled shRNA transduced cells $(\mathrm{n}=3)$ showed no breaks. After 4 weeks of LTC, we de- tected increased chromosome breakage in 6 out of $10 \mathrm{cul}-$ tures with a sufficient number of metaphases (at least 15 metaphases evaluable) ( 1 of 3 shRNA1/2, 2 of 3 scrambled, 3 of 4 mock). In 3 of the 6 cultures ( 1 shRNA1/2, 1 scrambled, 1 mock), 1 chromosome break was detected; in 1 (scrambled), 4 breaks occurred in 3 metaphases; in 1 (mock), 3 breaks occurred in 3 metaphases, and in 1 of the 6 cultures (mock), 2 breaks in 2 metaphases were detected.

In total, 17 break events occurred. Five breaks in $3 p, 3$ in $7 \mathrm{q}, 2$ in $4 \mathrm{q}$, and one break in 1p, 5q, 6q, 10q, 14q, 15q, and $\mathrm{Xq}$ each were detected. The following breakpoints occurred at common fragile sites: 3p14.2 (5×), 6q15, 7q21, $7 \mathrm{q} 32.3,10 \mathrm{q} 22$ and Xq27. Further breakpoints detected were: $1 \mathrm{p} 35,4 \mathrm{q} 22(2 \times), 5 \mathrm{q} 22,7 \mathrm{q} 31,14 \mathrm{q} 12$ and $5 \mathrm{q} 22$.

Surprisingly, after 42 days of culture, no further chromosomal breakage was detected ( 1 shRNA1/2, 2 scrambled, 4 mock cultures). All cells showed a normal stable karyotype. However, remarkably, in one LTC obtained from CD34+ cells transduced with scrambled shRNA, chromosomal breakage and single cell aberrations during the first 4 weeks of cultivation were followed by clonal dominance of monosomy 7 after 6 weeks, 45,XY,-7 [11]/46,XY[4] (fig. 4b). LM-PCR, however, did not detect integration into any common cancer-associated genes, e.g. EVI1.

\section{Telomere Length and TERT Expression in Long-Term Culture}

Interestingly, during culturing, the median telomere length decreased in RPS14-deficient cells as well as in control cells. Initially, telomeres showed a median length of about $11.94 \mathrm{~kb}$, decreasing to a median length of $9.0 \mathrm{~kb}$ $(\mathrm{n}=11$ different cultures, range $7.5-11.4 \mathrm{~kb})$ and $6.8 \mathrm{~kb}$ ( $\mathrm{n}=7$, range 4.7-9.0 kb) after 2 and 4 weeks, respectively, then after 6 weeks showing an apparent elongation to a level of $9.6 \mathrm{~kb}(\mathrm{n}=6$, range $7.8-11.5 \mathrm{~kb})$ (fig. 5).

To understand whether upregulation of telomerase was responsible for the observed apparent telomere lengthening, we measured TERT expression as a marker for telomerase activity during long-term culture on a weekly basis. However, TERT expression levels in follow-up showed no change from the level at time point 0 (data not shown).

\section{Discussion}

In summary, at the beginning of cell culture, CD34+ cells had a normal median telomere length of about $12 \mathrm{~kb}$. During follow-up, the median telomere length decreased and, simultaneously, an increased chromosomal instabil- 
Fig. 3. Number of $\gamma \mathrm{H} 2 \mathrm{AX}$ foci in RPS14deficient cells compared to control cells (scrambled and mock) with or without prior MMC treatment after $0,7,21,35$, and 49 days in long-term culture $(\mathbf{a}, \mathbf{b})$.
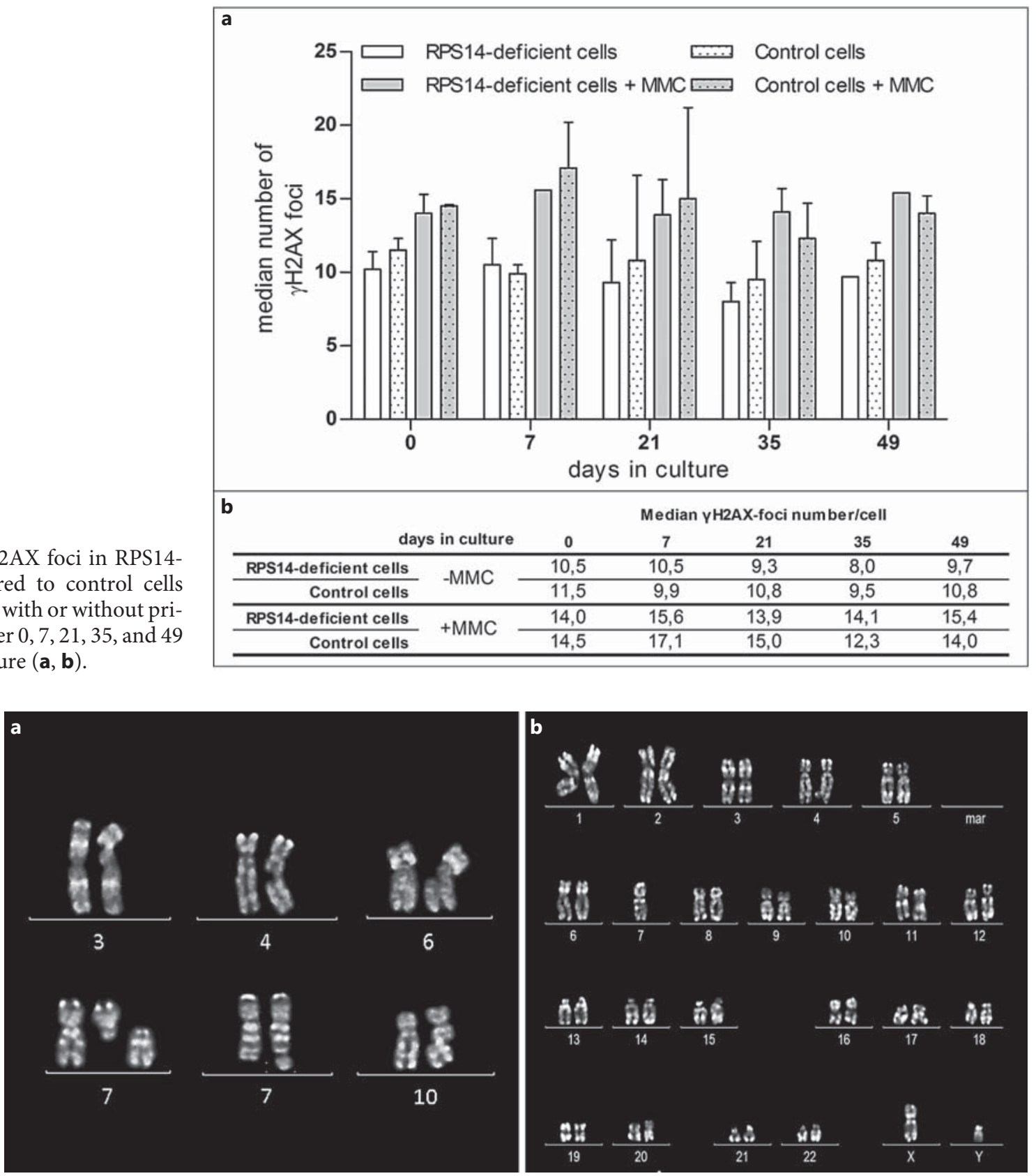

Fig. 4. a Chromosomes with nonclonal breaks indicating an increased chromosomal instability after 2-4 weeks of culture. b Karyogram (fluorescence R-banding) showing a clonal monosomy 7 in CD34+ cells derived from cord blood transduced with the scrambled shRNA vector after 4 weeks of culture.

ity could be detected - independent of prior transduction and RPS14 haploinsufficiency. The breakpoints involved no typical leukemic loci. However, 6 of 17 breaks occurred at fragile sites. The most common breakpoint at a fragile site was $3 p 14.2$. It occurred in 5 different cultures ( 2 scrambled, 3 mock) and is known as the 'most com- mon fragile site' in the genome [Durkin and Glover, 2007]. It is still not known whether breakages within those regions play a crucial role in leukemic development or whether certain loci, due to sequence characteristics like Alu repeats or fragile sites, are particularly prone to breakage [Ozeri-Galai et al., 2012]. During further cul- 

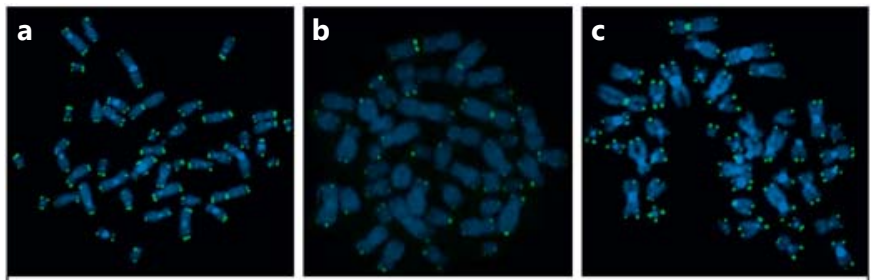

d

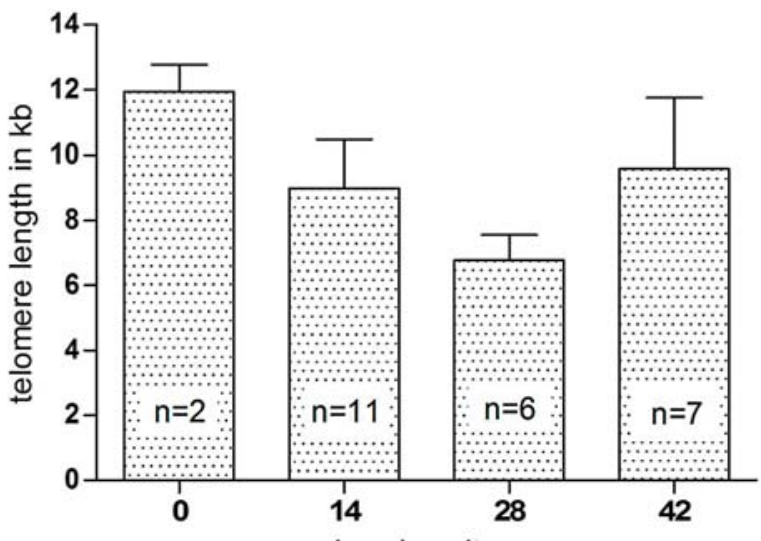

days in culture

Fig. 5. a T/C-FISH metaphase at the start of LTC (day 0). b At week 4 (mock). c At week 6 (mock). d Mean telomere length after 0, 14, 28 , and 42 days in long-term culture.

ture, it seemed that the telomeres re-elongated, and chromosomes also seemed to be stabilized again. TERT expression was not elevated.

Hematopoietic stem cells under proliferative stress, as for example after transplantation, have already been described to exhibit telomere shortening [Gadalla and Savage, 2011]. Other studies of HSCs have either been described not to show telomere shortening (after 7 days in short-term culture) [Andrade et al., 2010] or even an increase and a later decrease in telomere length in longterm culture [Gammaitoni et al., 2004]. Obviously, these results strongly depend on the source of HSCs and the respective cultivation protocol. In our study, HSCs experience excessive telomere shortening during proliferation and differentiation, independent of lentiviral transduction and knockdown of RPS14. Cells with critically short telomeres are known to be prone to chromosomal instability which we could visualize by chromosome breakage.

As in most cases, apoptosis is activated by short telomeres and chromosome breakage [Hanahan and Weinberg, 2011]. It is most likely that the cells have been strongly expanded in culture during the first weeks and, thus, have shortened telomeres, making them prone to chromosomal instability and leading to apoptosis after some weeks. Sub- sequently, other not yet excessively proliferating cells maintaining long telomeres and stable chromosomes might have experienced proliferative pressure and replenished the 'culture pool' with a population of cells with longer (or not yet shortened) telomeres. This 'cell selection'-effect explains why a decrease and then an apparent increase of telomere length was observed and also why chromosomes seem to stabilize again. This hypothesis might be underlined by a former study of individual CD34+ cells in cord blood that display extensive heterogeneity in growth kinetics [Bartolović et al., 2005]. In that study, it was shown that, among the highly proliferative clones, the most slowly growing clones were the ones with the longest telomeres.

Certainly, it would be very interesting to observe the CD34+ cells of our study for a longer time period. However, after 6 weeks, the number of RPS14- haploinsufficent cells quickly decreased, making further analyses with regard to our current research impossible. However, it occurred to us that other researchers, who do not regularly monitor cytogenetics and telomere length of their cultures, may have effects like this in their cultures of HSCs (and possibly other stem cell cultures) which may change their observations in such a manner that makes them hard to interpret.

Remarkably, one of our cultures (scrambled) even developed a stable clonal aberration, a monosomy 7 . The outgrowth of this clone demonstrates that cells with some certain aberrations, among those a loss of chromosome 7, are able to bypass apoptosis and senescence. Monosomy 7 is a characteristic aberration of myeloid neoplasia, especially MDS and acute myeloid leukemia.

In this model, haploinsufficiency of RPS14 led to a decreased erythroid differentiation capacity and a decreased proliferation. The other parameters observed (telomere length, cytogenetics and DNA repair) showed no change in comparison with the control cells, making it clear that a longer time period of observation, as in a xenograft mouse model, is needed.

Overall, LTC on feeder layer cells seems to be an appropriate system to analyze hematopoietic stem cells in culture for several weeks.

However, we learned here that, apart from insertional mutagenesis, cell culture artefacts inducing telomere shortening and chromosomal instability have to be taken into account and regular cytogenetic analyses should always be performed.

\section{Acknowledgement}

We thank Gillian Teicke for preparation of the manuscript. 


\section{References}

-Andrade PZ, dos Santos F, Almeida-Porada G, da Silva CL, S Cabral JM: Systematic delineation of optimal cytokine concentrations to expand hematopoietic stem/progenitor cells in $\mathrm{CO}_{-}$ culture with mesenchymal stem cells. Mol Biosyst 6:1207-1215 (2010).

-Bartolović K, Balabanov S, Berner B, Bühring HJ, Komor $\mathrm{M}$, et al: Clonal heterogeneity in growth kinetics of CD34+CD38- human cord blood cells in vitro is correlated with gene expression pattern and telomere length. Stem Cells 23:946-957 (2005).

Drexler HG, Dirks WG, Macleod RA: Many are called MDS cell lines: one is chosen. Leuk Res 33:1011-1016 (2009).

Durkin SG, Glover TW: Chromosome fragil sites. Annu Rev Genet 41:169-192 (2007).

Ebert BL, Pretz J, Bosco J, Chang CY, Tamayo P, et al: Identification of RPS14 as a 5q- syndrome gene by RNA interference screen. Nature 451:335-339 (2008).

Gadalla SM, Savage SA: Telomere biology in hematopoiesis and stem cell transplantation. Blood Rev 25:261-269 (2011).

- Gammaitoni L, Weisel KC, Gunetti M, Wu KD, Bruno $S$, et al: Elevated telomerase activity and minimal telomere loss in cord blood long-term cultures with extensive stem cell replication. Blood 103:4440-4448 (2004).

-Ge J, Cai H, Tan WS: Chromosomal stability during ex vivo expansion of UCB CD $34^{+}$cells. Cell Prolif 44:550-557 (2011).
Göhring G, Giagounidis A, Büsche G, Kreipe HH, Zimmermann $\mathrm{M}$, et al: Patients with $\operatorname{del}(5 \mathrm{q})$ MDS who fail to achieve sustained erythroid or cytogenetic remission after treatment with lenalidomide have an increased risk for clonal evolution and AML progression. Ann Hematol 89:365-374 (2010).

Göhring G, Lange K, Hofmann W, Nielsen KV, Hellström-Lindberg E, et al: Telomere shortening, clonal evolution and disease progression in myelodysplastic syndrome patients with $5 \mathrm{q}$ deletion treated with lenalidomide. Leukemia 26:356-358 (2012).

Hanahan D, Weinberg RA: Hallmarks of cancer: the next generation. Cell 144:646-674 (2011).

Hogge DE, Lansdorp PM, Reid D, Gerhard B, Eaves CJ: Enhanced detection, maintenance, and differentiation of primitive human hematopoietic cells in cultures containing murine fibroblasts engineered to produce human steel factor, interleukin-3, and granulocyte colony-stimulating factor. Blood 88:37653773 (1996).

ISCN (2009): An International System for $\mathrm{Hu}$ man Cytogenetic Nomenclature, Shaffer LG, Slovak ML, Campbell LJ (eds) (Karger, Basel 2009).

Jädersten M, Saft L, Smith A, Kulasekararaj A, Pomplun S, et al: TP53 mutations in low-risk myelodysplastic syndromes with $\operatorname{del}(5 q)$ predict disease progression. J Clin Oncol 29: 1971-1979 (2011).

Lange K, Holm L, Vang Nielsen K, Hahn A, Hofmann W, et al: Telomere shortening and chromosomal instability in myelodysplastic syndromes. Genes Chromosomes Cancer 49: 260-269 (2010).
Lefort N, Perrier AL, Laâbi Y, Varela C, Peschanski M: Human embryonic stem cells and genomic instability. Regen Med 4:899-909 (2009).

Moon SH, Kim JS, Park SJ, Lim JJ, Lee HJ, et al: Effect of chromosome instability on the maintenance and differentiation of human embryonic stem cells in vitro and in vivo. Stem Cell Res 6:50-59 (2011).

Ozeri-Galai E, Bester AC, Kerem B: The complex basis underlying common fragile site instability in cancer. Trends Genet 28:295-302 (2012).

Prockop DJ, Keating A: Relearning the lessons of genomic stability of human cells during expansion in culture: implications for clinical research. Stem Cells 30:1051-1052 (2012).

Ross AL, Leder DE, Weiss J, Izakovic J, Grichnik JM: Genomic instability in cultured stem cells: associated risks and underlying mechanisms. Regen Med 6:653-662 (2011).

Schlegelberger B, Metzke S, Harder S, ZühlkeJenisch R, Zhang Y, Siebert R: Classical and molecular cytogenetics of tumor cells, in Wegner RD (ed): Diagnostic Cytogenetics, pp 151-185 (Springer Verlag, Heidelberg 1999).

-Schmidt M, Hoffmann G, Wissler M, Lemke N, Müssig A, et al: Detection and direct genomic sequencing of multiple rare unknown flanking DNA in highly complex samples. Hum Gene Ther 12:743-749 (2001).

Tehranchi R, Woll PS, Anderson K, Buza-Vidas N, Mizukami T, et al: Persistent malignant stem cells in del $(5 q)$ myelodysplasia in remission. N Eng J Med 363:1025-1037 (2010). 